\title{
Approximation by modified Kantorovich-Szász type operators involving Charlier polynomials
}

\author{
K.J. Ansari ${ }^{1}$, M. Mursaleen ${ }^{2,3,4^{*}}$, M. Shareef KP5 and M. Ghouse ${ }^{6}$
}

\section{"Correspondence:}

mursaleenm@gmail.com

${ }^{2}$ Department of Medical Research,

China Medical University Hospital, China Medical University (Taiwan),

Taichung, Taiwan

${ }^{3}$ Department of Mathematics,

Aligarh Muslim University, Aligarh,

India

Full list of author information is

available at the end of the article

\begin{abstract}
In this paper, we give some direct approximation results by modified Kantorovich-Szász type operators involving Charlier polynomials. Further, approximation results are also developed in polynomial weighted spaces. Moreover, for the functions of bounded variation, approximation results are proved. Finally, some graphical examples are provided to show comparisons of convergence between old and modified operators towards a function under different parameters and conditions.
\end{abstract}

MSC: 41A10; 41A25; 41A36

Keywords: Szász operator; Charlier polynomials; Modulus of continuity; Rate of convergence

\section{Introduction}

In 1950, Szász [20] introduced positive linear operators in the sense of exponential growth on nonnegative semiaxes and exhaustively investigated them. These operators later became known as Szász operators. The Szász type operators involving Charlier polynomials were defined in [21] as

$$
L_{n}(f ; y, b)=e^{-1}\left(1-\frac{1}{b}\right)^{(b-1) n y} \sum_{l=0}^{\infty} \frac{C_{l}^{(b)}(-(b-1) n y)}{l !} f\left(\frac{l}{n}\right)
$$

where $b>1$ and $y \geq 0$, having the generating functions [5] of the form

$$
e^{t}\left(1-\frac{t}{b}\right)^{u}=\sum_{l=0}^{\infty} C_{l}^{(b)}(u) \frac{t^{l}}{l !}, \quad|t|<b
$$

where $C_{l}^{(b)}(u)=\sum_{r=0}^{l}\left(\begin{array}{l}l \\ r\end{array}\right)(-u)_{r}\left(\frac{1}{b}\right)^{r}$ and $(k)_{0}=1,(k)_{m}=k(k+1) \cdots(k+m-1)$, for $m \geq 1$.

(c) The Author(s) 2020. This article is licensed under a Creative Commons Attribution 4.0 International License, which permits use, sharing, adaptation, distribution and reproduction in any medium or format, as long as you give appropriate credit to the original author(s) and the source, provide a link to the Creative Commons licence, and indicate if changes were made. The images or other third party material in this article are included in the article's Creative Commons licence, unless indicated otherwise in a credit line to the material. If material is not included in the article's Creative Commons licence and your intended use is not permitted by statutory regulation or exceeds the permitted use, you will need to obtain permission directly from the copyright holder. To view a copy of this licence, visit http://creativecommons.org/licenses/by/4.0/. 
Motivated by the work done in [9], we define the Kantorovich generalization [10] of (1.2) as follows:

$$
\mathcal{Q}_{n, b}^{\left(\mu_{n}, v_{n}\right)}(f ; y)=v_{n} e^{-1}\left(1-\frac{1}{b}\right)^{(b-1) \mu_{n} y} \sum_{l=0}^{\infty} \frac{C_{l}^{(b)}\left(-(b-1) \mu_{n} y\right)}{l !} \int_{l / v_{n}}^{l+1 / v_{n}} f(t) d t
$$

where $\mu_{n}$ and $v_{n}$ are sequences of positive numbers which are increasing and unbounded such that

$$
\lim _{n \rightarrow \infty} \frac{1}{v_{n}}=0, \quad \frac{\mu_{n}}{v_{n}}=1+O\left(\frac{1}{v_{n}}\right)
$$

If we take $\mu_{n}=v_{n}=n$, we will have the operators defined in [9].

For some recent and interesting results on the various generalizations and corresponding approximation results, we refer to $[1,3,6-8,14-17,22]$.

\section{Auxiliary results}

We first present some auxiliary results.

Lemma 2.1 Let $\mathcal{Q}_{n, b}^{\left(\mu_{n}, v_{n}\right)}$ be defined by (1.3). Then, we have

1. $\mathcal{Q}_{n, b}^{\left(\mu_{n}, v_{n}\right)}(1 ; y)=1$,

2. $\mathcal{Q}_{n, b}^{\left(\mu_{n}, v_{n}\right)}(t ; y)=\frac{\mu_{n}}{v_{n}} y+\frac{3}{2 v_{n}}$

3. $\mathcal{Q}_{n, b}^{\left(\mu_{n}, v_{n}\right)}\left(t^{2} ; y\right)=\frac{\mu_{n}^{2}}{v_{n}^{2}} y^{2}+\frac{\mu_{n}}{v_{n}^{2}}\left(4+\frac{1}{b-1}\right) y+\frac{10}{3 v_{n}^{2}}$.

4. $\mathcal{Q}_{n, b}^{\left(\mu_{n}, v_{n}\right)}\left(t^{3} ; y\right)=\frac{\mu_{n}^{3}}{v_{n}^{3}} y^{3}+\frac{\mu_{n}^{2}}{v_{n}^{3}}\left(\frac{15}{2}+\frac{3}{b-1}\right) y^{2}+\frac{\mu_{n}}{v_{n}^{3}}\left(\frac{31}{2}+\frac{15}{2(b-1)}+\frac{2}{(b-1)^{2}}\right) y+\frac{37}{4 v_{n}^{3}}$.

5. $\mathcal{Q}_{n, b}^{\left(\mu_{n}, v_{n}\right)}\left(t^{4} ; y\right)=$

$$
\frac{\mu_{n}^{4}}{v_{n}^{4}} y^{4}+\frac{\mu_{n}^{3}}{v_{n}^{4}}\left(12+\frac{6}{b-1}\right) y^{3}+\frac{\mu_{n}^{2}}{v_{n}^{4}}\left(46+\frac{36}{b-1}+\frac{11}{(b-1)^{2}}\right) y^{2}+\frac{\mu_{n}}{v_{n}^{4}}\left(64+\frac{46}{b-1}+\frac{24}{(b-1)^{2}}+\frac{6}{(b-1)^{3}}\right) y+\frac{151}{5 v_{n}^{4}} \text {. }
$$

Proof With the help of the Charlier polynomials' generating function given by (1.2), after some simple calculations, we obtain

$$
\begin{aligned}
\sum_{l=0}^{\infty} \frac{C_{l}^{(b)}\left(-(b-1) \mu_{n} y\right)}{l !}=e\left(1-\frac{1}{b}\right)^{-(b-1) \mu_{n} y}, & \\
\sum_{l=0}^{\infty} \frac{l C_{l}^{(b)}\left(-(b-1) \mu_{n} y\right)}{l !}= & e\left(1-\frac{1}{b}\right)^{-(b-1) \mu_{n} y}\left(1+\mu_{n} y\right), \\
\sum_{l=0}^{\infty} \frac{l^{2} C_{l}^{(b)}\left(-(b-1) \mu_{n} y\right)}{l !}= & e\left(1-\frac{1}{b}\right)^{-(b-1) \mu_{n} y}\left(\mu_{n}^{2} y^{2}+\mu_{n} y\left(3+\frac{1}{b-1}\right)+2\right), \\
\sum_{l=0}^{\infty} \frac{l^{3} C_{l}^{(b)}\left(-(b-1) \mu_{n} y\right)}{l !}= & e\left(1-\frac{1}{b}\right)^{-(b-1) \mu_{n} y}\left(\mu_{n}^{3} y^{3}+\mu_{n}^{2}\left(6+\frac{3}{b-1}\right) y^{2}\right. \\
& \left.+\mu_{n}\left(10+\frac{6}{b-1}+\frac{2}{(b-1)^{2}}\right) y+5\right),
\end{aligned}
$$




$$
\begin{aligned}
\sum_{l=0}^{\infty} \frac{l^{4} C_{l}^{(b)}\left(-(b-1) \mu_{n} y\right)}{l !}= & e\left(1-\frac{1}{b}\right)^{-(b-1) \mu_{n} y}\left(\mu_{n}^{4} y^{4}+\mu_{n}^{3}\left(10+\frac{6}{b-1}\right) y^{3}\right. \\
& +\mu_{n}^{2}\left(32+\frac{30}{b-1}+\frac{11}{(b-1)^{2}}\right) y^{2} \\
& \left.+\mu_{n}\left(37+\frac{32}{b-1}+\frac{20}{(b-1)^{2}}+\frac{6}{(b-1)^{3}}\right) y+15\right) .
\end{aligned}
$$

From the above equalities, the claims of the lemma can be obtained.

Lemma 2.2 For the operator $\mathcal{Q}_{n, b}^{\left(\mu_{n}, v_{n}\right)}$ given by (1.3), we have the following equalities:

$$
\begin{aligned}
& \text { (i) } \mathcal{Q}_{n, b}^{\left(\mu_{n}, v_{n}\right)}(t-y ; y)=\left(\frac{\mu_{n}}{v_{n}}-1\right) y+\frac{1}{v_{n}}, \\
& \text { (ii) } \mathcal{Q}_{n, b}^{\left(\mu_{n}, v_{n}\right)}\left((t-y)^{2} ; y\right)=\left(\frac{\mu_{n}}{v_{n}}-1\right)^{2} y^{2}+\left(\frac{\mu_{n}}{v_{n}^{2}}\left(3+\frac{1}{b-1}\right)-\frac{2}{v_{n}}\right) y+\frac{2}{v_{n}^{2}}, \\
& \text { (iii) } \mathcal{Q}_{n, b}^{\left(\mu_{n}, v_{n}\right)}\left((t-y)^{4} ; y\right) \\
& =\left(\frac{\mu_{n}}{v_{n}}-1\right) y^{4} \\
& \quad+2\left(\frac{\mu_{n}^{3}}{v_{n}^{4}}\left(5+\frac{3}{b-1}\right)-\frac{6 \mu_{n}^{2}}{v_{n}^{3}}\left(2+\frac{1}{b-1}\right)+\frac{3 \mu_{n}}{v_{n}^{2}}\left(3+\frac{1}{b-1}\right)-\frac{2}{v_{n}}\right) y^{3} \\
& \quad+\left(\frac{\mu_{n}^{2}}{v_{n}^{4}}\left(32+\frac{30}{b-1}+\frac{11}{(b-1)^{2}}\right)-\frac{4 \mu_{n}}{v_{n}^{3}}\left(10+\frac{6}{b-1}+\frac{2}{(b-1)^{2}}\right)+\frac{12}{v_{n}^{2}}\right) y^{2} \\
& \quad+\left(\frac{\mu_{n}}{v_{n}^{4}}\left(37+\frac{32}{b-1}+\frac{20}{(b-1)^{2}}+\frac{6}{(b-1)^{3}}\right)-\frac{20}{v_{n}^{3}}\right) y+\frac{15}{v_{n}^{4}} .
\end{aligned}
$$

\section{Local approximation results}

In what follows, let $\mathcal{Q}_{n, b}^{\left(\mu_{n}, v_{n}\right)}(t-y ; y)=\chi_{\mu_{n}, v_{n}}(y)$ and $\mathcal{Q}_{n, b}^{\left(\mu_{n}, v_{n}\right)}\left((t-y)^{2} ; y\right)=\xi_{\mu_{n}, v_{n}}(y)$. We will now give two theorems on the uniform convergence and the order of approximation.

Theorem 3.1 Let $f \in C[0, \infty) \cap G$. Then $\lim _{n \rightarrow \infty} \mathcal{Q}_{n, b}^{\left(\mu_{n}, v_{n}\right)}(f ; y)=f(y)$, the sequence of operators Eq. (1.3) converges uniformly in each compact subset of $[0, \infty)$, where

$$
G:=\left\{f: \mathbb{R}_{0}^{+} \rightarrow \mathbb{R},|F(y)|=\left|\int_{0}^{y} f(s) d s\right| \leq K e^{B y}, K \in \mathbb{R}^{+} \text {and } B \in \mathbb{R}\right\} .
$$

Proof From Lemma 2.1(1)-(3), we get

$$
\lim _{n \rightarrow \infty} \mathcal{Q}_{n, b}^{\left(\mu_{n}, v_{n}\right)}\left(s^{k} ; y\right)=y^{k}, \quad k=0,1,2 .
$$

The proof of the theorem is established by taking advantage of the above uniform convergence in each compact subset of $[0, \infty)$ and the famous Korovkin's theorem.

Suppose $f \in \tilde{C}[0, \infty)$, i.e., $f$ belongs to the space of uniformly continuous functions on $[0, \infty)$. If $\delta>0$, then the modulus of continuity $\omega(f, \delta)$ is defined by

$$
\omega(f, \delta):=\sup _{\substack{\sigma, \varsigma \in[0, \infty) \\|\sigma-\varsigma| \leq \delta}}|f(\sigma)-f(\varsigma)| .
$$


Theorem 3.2 Let $f \in \tilde{C}[0, \infty) \cap$ E. For the operators $\mathcal{Q}_{n, b}^{\left(\mu_{n}, v_{n}\right)}(f ; y)$ given by $(1.3)$ the following estimate holds:

$$
\begin{aligned}
& \left|\mathcal{Q}_{n, b}^{\left(\mu_{n}, v_{n}\right)}(f ; y)-f(y)\right| \\
& \quad \leq\left\{1+\sqrt{\left(\mu_{n}-v_{n}\right)^{2} y^{2}+\left(\left(4+\frac{1}{b-1}\right) \mu_{n}-3 v_{n}\right) y+\frac{10}{3}}\right\} \omega\left(f, \frac{1}{v_{n}}\right) .
\end{aligned}
$$

Proof From (1.3) and the property of modulus of continuity, the left-hand side of (3.1) leads to

$$
\begin{aligned}
& \left|\mathcal{Q}_{n, b}^{\left(\mu_{n}, v_{n}\right)}(f ; y)-f(y)\right| \\
& \quad \leq v_{n} e^{-1}\left(1-\frac{1}{b}\right)^{(b-1) \mu_{n} y} \sum_{l=0}^{\infty} \frac{C_{l}^{(b)}\left(-(b-1) \mu_{n} y\right)}{l !} \int_{l / v_{n}}^{l+1 / v_{n}}|f(t)-f(y)| d t \\
& \quad \leq\left\{1+\frac{1}{\delta} v_{n} e^{-1}\left(1-\frac{1}{b}\right)^{(b-1) \mu_{n} y} \sum_{l=0}^{\infty} \frac{C_{l}^{(b)}\left(-(b-1) \mu_{n} y\right)}{l !} \int_{l / v_{n}}^{l+1 / v_{n}}|t-y| d t\right\} \omega(f, \delta) .
\end{aligned}
$$

Using Cauchy-Schwarz inequality for the integral, we get

$$
\begin{aligned}
& \left|\mathcal{Q}_{n, b}^{\left(\mu_{n}, v_{n}\right)}(f ; y)-f(y)\right| \\
& \leq\left\{1+\frac{1}{\delta} e^{-1}\left(1-\frac{1}{b}\right)^{(b-1) \mu_{n} y}\right. \\
& \left.\quad \times \sum_{l=0}^{\infty} \frac{C_{l}^{(b)}\left(-(b-1) \mu_{n} y\right)}{l !}\left(v_{n} \int_{l / v_{n}}^{l+1 / v_{n}}(t-y)^{2} d t\right)^{1 / 2}\right\} \omega(f, \delta) .
\end{aligned}
$$

In the above sum, we apply Cauchy-Schwarz inequality, and then in view of Lemma 2.1, (3.2) becomes

$$
\begin{aligned}
\left|\mathcal{Q}_{n, b}^{\left(\mu_{n}, v_{n}\right)}(f ; y)-f(y)\right| & \\
\leq & \left\{1+\frac{1}{\delta}\left(e^{-1}\left(1-\frac{1}{b}\right)^{(b-1) \mu_{n} y} \sum_{l=0}^{\infty} \frac{C_{l}^{(b)}\left(-(b-1) \mu_{n} y\right)}{l !}\right)^{1 / 2}\right. \\
& \left.\times\left(v_{n} e^{-1}\left(1-\frac{1}{b}\right)^{(b-1) \mu_{n} y} \sum_{l=0}^{\infty} \frac{C_{l}^{(b)}\left(-(b-1) \mu_{n} y\right)}{l !} \int_{l / v_{n}}^{l+1 / v_{n}}(t-y)^{2} d t\right)^{1 / 2}\right\} \omega(f, \delta) \\
\leq & \left\{1+\frac{1}{\delta}\left(\mathcal{Q}_{n, b}^{\left(\mu_{n}, v_{n}\right)}\left((t-y)^{2} ; y, b\right)\right)^{1 / 2}\right\} \omega(f, \delta) \\
\leq & \left\{1+\frac{1}{\delta} \frac{1}{v_{n}} \sqrt{\left(\mu_{n}-v_{n}\right)^{2} y^{2}+\left(\left(4+\frac{1}{b-1}\right) \mu_{n}-3 v_{n}\right) y+\frac{10}{3}}\right\} \omega\left(f, \frac{1}{\delta}\right),
\end{aligned}
$$

where, taking $\delta=\frac{1}{v_{n}}$, we get (3.1). 
Let $a_{1}, a_{2}>0$ be fixed. We now consider the following space of Lipschitz type (see [18]):

$$
\operatorname{Lip}_{M}^{\left(a_{1}, a_{2}\right)}(r):=\left\{f \in C[0, \infty):|f(\kappa)-f(\lambda)| \leq M \frac{|\kappa-\lambda|^{r}}{\left(\kappa+a_{1} \lambda^{2}+a_{2} \lambda\right)^{r / 2}} ; \lambda, \kappa>0\right\}
$$

where $M$ is a positive constant and $0<r \leq 1$.

Theorem 3.3 Let $f \in \operatorname{Lip}_{M}^{\left(a_{1}, a_{2}\right)}(r)$ and $r \in(0,1]$, then $\forall y>0$, we have

$$
\left|\mathcal{Q}_{n, b}^{\left(\mu_{n}, v_{n}\right)}(f ; y)-f(y)\right| \leq M\left(\frac{\xi_{\mu_{n}, v_{n}}(y)}{\left(a_{1} y^{2}+a_{2} y\right)}\right)^{r / 2} .
$$

Proof Since

$$
|f(t)-f(y)| \leq M \frac{|t-y|^{r}}{\left(t+a_{1} y^{2}+a_{2} y\right)^{r / 2}},
$$

one has

$$
\left|\mathcal{Q}_{n, b}^{\left(\mu_{n}, v_{n}\right)}(f ; y)-f(y)\right| \leq M \mathcal{Q}_{n, b}^{\left(\mu_{n}, v_{n}\right)}\left(\frac{|t-y|^{r}}{\left(t+a_{1} y^{2}+a_{2} y\right)^{r / 2}} ; y\right) .
$$

Applying Hölder's inequality with $p=\frac{2}{r}$ and $\frac{2}{2-r}$, we find that

$$
\left|\mathcal{Q}_{n, b}^{\left(\mu_{n}, v_{n}\right)}(f ; y)-f(y)\right| \leq M \mathcal{Q}_{n, b}^{\left(\mu_{n}, v_{n}\right)}\left(\frac{(t-y)^{2}}{t+a_{1} y^{2}+a_{2} y} ; y\right)^{r / 2} .
$$

Since $f \in \operatorname{Lip}_{M}^{\left(a_{1}, a_{2}\right)}(r)$ and $\frac{1}{t+a_{1} y^{2}+a_{2} y}<\frac{1}{a_{1} y^{2}+a_{2} y}, \forall y \in(0, \infty)$, we have

$$
\begin{aligned}
\left|\mathcal{Q}_{n, b}^{\left(\mu_{n}, v_{n}\right)}(f ; y)-f(y)\right| & \leq M \mathcal{Q}_{n, b}^{\left(\mu_{n}, v_{n}\right)}\left(\frac{(t-y)^{2}}{a_{1} y^{2}+a_{2} y} ; y\right)^{r / 2} \\
& \leq \frac{M}{\left(a_{1} y^{2}+a_{2} y\right)^{r / 2}} \mathcal{Q}_{n, b}^{\left(\mu_{n}, v_{n}\right)}\left((t-y)^{2} ; y\right)^{r / 2} \\
& =M\left(\frac{\xi_{\mu_{n}, v_{n}}(y)}{\left(a_{1} y^{2}+a_{2} y\right)}\right)^{r / 2} .
\end{aligned}
$$

Our proof is now completed.

We denote the space of all functions $h$ on $[0,1)$ which are real-valued, uniformly continuous, as well as bounded by $\tilde{C}_{B}[0, \infty)$ and endow it with the norm $\|h\|_{\infty}=\sup _{y \in[0,1)}|h(y)|$. Further, we obtain a local direct estimate for the operators (1.3), using the Lipschitz maximal function of order $r$ introduced by Lenze [13] as:

$$
\tilde{\omega}_{r}(h, y)=\sup _{t \neq y, t \in[0, \infty)} \frac{|h(t)-h(y)|}{|t-y|^{r}}
$$

where $y \in[0,1)$ and $r \in(0,1]$. 
Theorem 3.4 Let $f \in \tilde{C}_{B}[0, \infty)$ and $0<r \leq 1$, then $\forall y \in[0, \infty)$

$$
\left|\mathcal{Q}_{n, b}^{\left(\mu_{n}, v_{n}\right)}(f ; y)-f(y)\right| \leq \tilde{\omega}_{r}(f, y)\left(\xi_{\mu_{n}, v_{n}}(y)\right)^{r} .
$$

Proof By equation (3.4),

$$
|f(t)-f(y)| \leq \tilde{\omega}_{r}(f, y)|t-y|^{r} .
$$

Applying $\mathcal{Q}_{n, b}^{\left(\mu_{n}, v_{n}\right)}$ on both sides of the above inequality, then using Lemma 2.1, as well as Hölder's inequality with $p=2 / r, q=2 /(2-r)$, we obtain

$$
\begin{aligned}
\left|\mathcal{Q}_{n, b}^{\left(\mu_{n}, v_{n}\right)}(f ; y)-f(y)\right| & \leq \tilde{\omega}_{r}(f, y) \mathcal{Q}_{n, b}^{\left(\mu_{n}, v_{n}\right)}\left(|t-y|^{r} ; y\right) \\
& \leq \tilde{\omega}_{r}(f, y)\left(\mathcal{Q}_{n, b}^{\left(\mu_{n}, v_{n}\right)}\left((t-y)^{2} ; y\right)\right)^{r / 2} \\
& =\tilde{\omega}_{r}(f, y)\left(\xi_{\mu_{n}, v_{n}}(y)\right)^{r / 2} .
\end{aligned}
$$

Thus, we have our desired result.

The Peetre's $K$-functional is given by

$$
K(g, \delta)=\inf _{h \in \tilde{C}_{B}^{2}[0, \infty)}\left\{\|g-h\|_{\infty}+\delta\|h\|_{\tilde{C}_{B}^{2}}\right\},
$$

where $\tilde{C}_{B}^{2}[0, \infty)=\left\{h \in \tilde{C}_{B}[0, \infty): h^{\prime}, h^{\prime \prime} \in \tilde{C}_{B}[0, \infty)\right\}$ with the norm $\|h\|_{\tilde{C}_{B}^{2}}=\|h\|_{\infty}+\left\|h^{\prime}\right\|_{\infty}+$ $\left\|h^{\prime \prime}\right\|_{\infty}$. Also, the inequality

$$
K(g, \delta) \leq M\left(\omega_{2}(g, \sqrt{\delta})+\min \{1, \delta\}\|g\|_{\infty}\right)
$$

holds for all $\delta>0$, where $\omega_{2}$ is the second-order modulus of smoothness of $g \in \tilde{C}_{B}[0, \infty)$, which is defined by

$$
\omega_{2}(g, \delta)=\sup _{0<|h| \leq \delta y, y+2 h \in[0, \infty)} \sup _{0}|g(y+2 h)-2 g(y+h)+g(y)| .
$$

Theorem 3.5 Iff $\in \tilde{C}_{B}[0, \infty)$, then

$$
\left|\mathcal{Q}_{n, b}^{\left(\mu_{n}, v_{n}\right)}(f ; y)-f(y)\right| \leq 4 K\left(f, \zeta_{\mu_{n}, v_{n}}(y)\right)+\omega\left(f, \chi_{\mu_{n}, v_{n}}(y)\right),
$$

where $\zeta_{\mu_{n}, v_{n}}(y)=\left(\xi_{\mu_{n}, v_{n}}(y)+\chi_{\mu_{n}, v_{n}}^{2}(y)\right) / 4$. Furthermore,

$$
\left|\mathcal{Q}_{n, b}^{\left(\mu_{n}, v_{n}\right)}(f ; y)-f(y)\right| \leq M\left(\omega_{2}\left(f, \sqrt{\zeta_{\mu_{n}, v_{n}}(y)}\right)+\min \left\{1, \zeta_{\mu_{n}, v_{n}}(y)\right\}\|f\|_{\infty}\right)+\omega\left(f,\left|\chi_{\mu_{n}, v_{n}}(y)\right|\right)
$$

Proof For $f \in \tilde{C}_{B}[0, \infty)$, we define the auxiliary operator as follows:

$$
\tilde{\mathcal{Q}}_{n, b}^{\left(\mu_{n}, v_{n}\right)}(f ; y)=\mathcal{Q}_{n, b}^{\left(\mu_{n}, v_{n}\right)}(f ; y)-f\left(\frac{\mu_{n}}{v_{n}} y+\frac{3}{2 v_{n}}\right)+f(y) .
$$


After taking the absolute value of both sides,

$$
\begin{aligned}
\left|\tilde{\mathcal{Q}}_{n, b}^{\left(\mu_{n}, v_{n}\right)}(f ; y)\right| & \leq\left|\mathcal{Q}_{n, b}^{\left(\mu_{n}, v_{n}\right)}(f ; y)\right|+\left|f\left(\frac{\mu_{n}}{v_{n}} y+\frac{3}{2 v_{n}}\right)\right|+|f(y)| \\
& \leq\|f\|_{\infty}\left|\mathcal{Q}_{n, b}^{\left(\mu_{n}, v_{n}\right)}(1 ; y, b)\right|+\|f\|_{\infty}+\|f\|_{\infty} \\
& \leq 3\|f\|_{\infty} .
\end{aligned}
$$

By Lemma 2.1, we have $\tilde{\mathcal{Q}}_{n, b}^{\left(\mu_{n}, v_{n}\right)}(t ; y)=y$, and therefore $\tilde{\mathcal{Q}}_{n, b}^{\left(\mu_{n}, v_{n}\right)}(t-y ; y)=0$.

Let $g \in \tilde{C}_{B}^{2}[0, \infty)$, using Taylor's theorem, we can write

$$
g(t)=g(y)+g^{\prime}(y)(t-y)+\int_{y}^{t}(t-u) g^{\prime \prime}(u) d u
$$

Applying operator $\tilde{\mathcal{Q}}_{n, b}^{\left(\mu_{n}, v_{n}\right)}$ to the above equation, we get

$$
\begin{aligned}
& \tilde{\mathcal{Q}}_{n, b}^{\left(\mu_{n}, v_{n}\right)}(g ; y)-g(y) \\
& \quad=\tilde{\mathcal{Q}}_{n, b}^{\left(\mu_{n}, v_{n}\right)}\left(\int_{y}^{t}(t-u) g^{\prime \prime}(u) d u ; y\right) \\
& \quad=\mathcal{Q}_{n, b}^{\left(\mu_{n}, v_{n}\right)}\left(\int_{y}^{t}(t-u) g^{\prime \prime}(u) d u ; y\right)-\int_{y}^{\frac{\mu_{n}}{v_{n}} y+\frac{3}{2 v_{n}}}\left(\frac{\mu_{n}}{v_{n}} y+\frac{3}{2 v_{n}}-u\right) g^{\prime \prime}(u) d u .
\end{aligned}
$$

Now taking the absolute value of both sides, we obtain

$$
\begin{aligned}
& \left|\tilde{\mathcal{Q}}_{n, b}^{\left(\mu_{n}, v_{n}\right)}(g ; y)-g(y)\right| \\
& \quad \leq\left|\mathcal{Q}_{n, b}^{\left(\mu_{n}, v_{n}\right)}\left(\int_{y}^{t}(t-u) g^{\prime \prime}(u) d u ; y\right)\right|+\left|\int_{y}^{\frac{\mu_{n}}{v_{n}} y+\frac{3}{2 v_{n}}}\left(\frac{\mu_{n}}{v_{n}} y+\frac{3}{2 v_{n}}-u\right) g^{\prime \prime}(u) d u\right| \\
& \quad \leq \mathcal{Q}_{n, b}^{\left(\mu_{n}, v_{n}\right)}\left(\left|\int_{y}^{t}\right|(t-u)|| g^{\prime \prime}(u)|d u| ; y\right)+\left|\left(\int_{y}^{\frac{\mu_{n}}{v_{n}} y+\frac{3}{2 v_{n}}}\left|\frac{\mu_{n}}{v_{n}} y+\frac{3}{2 v_{n}}-u\right|\left|g^{\prime \prime}(u)\right| d u\right)\right| \\
& \quad \leq\left\|g^{\prime \prime}\right\|_{\infty}\left\{\mathcal{Q}_{n, b}^{\left(\mu_{n}, v_{n}\right)}\left(\left|\int_{y}^{t}\right|(t-u)|d u| ; y\right)+\left|\left(\int_{y}^{\frac{\mu_{n}}{v_{n}} y+\frac{3}{2 v_{n}}}\left|\frac{\mu_{n}}{v_{n}} y+\frac{3}{2 v_{n}}-u\right| d u\right)\right|\right\} .
\end{aligned}
$$

Therefore, by using the norm on $g$, we have

$$
\begin{aligned}
\left|\tilde{\mathcal{Q}}_{n, b}^{\left(\mu_{n}, v_{n}\right)}(g ; y)-g(y)\right| & \leq\|g\|_{\tilde{C}_{B}^{2}}\left\{\mathcal{Q}_{n, b}^{\left(\mu_{n}, v_{n}\right)}\left((t-y)^{2} ; y\right)+\left(\frac{\mu_{n}}{v_{n}} y+\frac{3}{2 v_{n}}-y\right)^{2}\right\} \\
& \leq\|g\|_{\tilde{C}_{B}^{2}}\left\{\mathcal{Q}_{n, b}^{\left(\mu_{n}, v_{n}\right)}\left((t-y)^{2} ; y\right)+\left(\mathcal{Q}_{n, b}^{\left(\mu_{n}, v_{n}\right)}(t-y ; y)\right)^{2}\right\} \\
& \leq\|g\|_{\tilde{C}_{B}^{2}}\left\{\xi_{\mu_{n}, v_{n}}(y)+\chi_{\mu_{n}, v_{n}}^{2}(y)\right\} .
\end{aligned}
$$

Now, using the definition of auxiliary operators (3.5), we get

$$
\begin{aligned}
& \left|\mathcal{Q}_{n, b}^{\left(\mu_{n}, v_{n}\right)}(f ; y)-f(y)\right| \\
& \quad \leq\left|\tilde{\mathcal{Q}}_{n, b}^{\left(\mu_{n}, v_{n}\right)}(f ; y)-f(y)+f\left(\frac{\mu_{n}}{v_{n}} y+\frac{3}{2 v_{n}}\right)-f(y)\right|
\end{aligned}
$$




$$
\begin{aligned}
\leq & \left|\tilde{\mathcal{Q}}_{n, b}^{\left(\mu_{n}, v_{n}\right)}(f-g ; y)\right|+\left|\tilde{\mathcal{Q}}_{n, b}^{\left(\mu_{n}, v_{n}\right)}(g ; y)-g(y)\right| \\
& +|g(y)-f(y)|+\left|f\left(\frac{\mu_{n}}{v_{n}} y+\frac{3}{2 v_{n}}\right)-f(y)\right| .
\end{aligned}
$$

Combining (3.6) and (3.7) with the above equation, we get

$$
\begin{aligned}
& \left|\mathcal{Q}_{n, b}^{\left(\mu_{n}, v_{n}\right)}(f ; y)-f(y)\right| \\
& \quad \leq 3\|f-g\|_{\infty}+\|g\|_{\tilde{C}_{B}^{2}}\left\{\xi_{\mu_{n}, v_{n}}(y)+\chi_{\mu_{n}, v_{n}}^{2}(y)\right\}+\|f-g\|_{\infty}+\omega\left(f,\left|\frac{\mu_{n}}{v_{n}} y+\frac{3}{2 v_{n}}-y\right|\right) \\
& \quad \leq 4\|f-g\|_{\infty}+\|g\|_{\tilde{C}_{B}^{2}} 4 \zeta_{\mu_{n}, v_{n}}(y)+\omega\left(f,\left|\chi_{\mu_{n}, v_{n}}(y)\right|\right),
\end{aligned}
$$

and after taking the infimum on the right-hand side over all $g \in \tilde{C}_{B}^{2}$, we have

$$
\begin{aligned}
& \left|\mathcal{Q}_{n, b}^{\left(\mu_{n}, v_{n}\right)}(f ; y)-f(y)\right| \\
& \quad \leq 4 K\left(f, \zeta_{\mu_{n}, v_{n}}(y)\right)+\omega\left(f,\left|\chi_{\mu_{n}, v_{n}}(y)\right|\right) \\
& \quad \leq M\left(\omega_{2}\left(f, \sqrt{\zeta_{\mu_{n}, v_{n}}(y)}\right)+\min \left\{1, \zeta_{\mu_{n}, v_{n}}(y)\right\}\|f\|_{\infty}\right)+\omega\left(f,\left|\chi_{\mu_{n}, v_{n}}(y)\right|\right) .
\end{aligned}
$$

This completes the proof of the theorem.

Theorem 3.6 Let $f \in \tilde{C}_{B}^{1}[0, \infty)$, then $\forall y \geq 0$ and $\delta>0$,

$$
\left|\mathcal{Q}_{n, b}^{\left(\mu_{n}, v_{n}\right)}(f ; y)-f(y)\right| \leq\left\{\left|f^{\prime}(y)\right|+2 \omega\left(f^{\prime}, \delta_{n}(y)\right)\right\} .
$$

Proof Since $f \in \tilde{C}_{B}^{1}[0, \infty)$, we can write

$$
f(t)-f(y)=f^{\prime}(y)(t-y)+\int_{y}^{t}\left(f^{\prime}(u)-f^{\prime}(y)\right) d u .
$$

Now, using the well-known property of the modulus of continuity for $\delta>0$ and $f \in$ $\tilde{C}_{B}^{1}[0, \infty)$,

$$
\left|f^{\prime}(u)-f^{\prime}(y)\right| \leq\left(\frac{|u-y|}{\delta}+1\right) \omega\left(f^{\prime}, \delta\right),
$$

hence

$$
\left|\int_{y}^{t}\left(f^{\prime}(u)-f^{\prime}(y)\right) d u\right| \leq\left(\frac{(t-y)^{2}}{\delta}+|t-y|\right) \omega\left(f^{\prime}, \delta\right)
$$

Therefore, from (3.8) and the above equation, we have

$$
\begin{aligned}
& \left|\mathcal{Q}_{n, b}^{\left(\mu_{n}, v_{n}\right)}(f ; y)-f(y)\right| \\
& \quad \leq\left|f^{\prime}(y)\right| \mathcal{Q}_{n, b}^{\left(\mu_{n}, v_{n}\right)}(|t-y| ; y)+\left(\frac{1}{\delta} \mathcal{Q}_{n, b}^{\left(\mu_{n}, v_{n}\right)}\left((t-y)^{2} ; y\right)+\mathcal{Q}_{n, b}^{\left(\mu_{n}, v_{n}\right)}(|t-y| ; y)\right) \omega\left(f^{\prime}, \delta\right) .
\end{aligned}
$$


After applying the Cauchy-Schwarz inequality, we get

$$
\begin{aligned}
& \left|\mathcal{Q}_{n, b}^{\left(\mu_{n}, v_{n}\right)}(f ; y)-f(y)\right| \\
& \leq \quad\left(\left|f^{\prime}(y)\right|+\omega\left(f^{\prime}, \delta\right)\right) \sqrt{\mathcal{Q}_{n, b}^{\left(\mu_{n}, v_{n}\right)}\left((t-y)^{2} ; y\right)} \sqrt{\mathcal{Q}_{n, b}^{\left(\mu_{n}, v_{n}\right)}(1 ; y)} \\
& \quad+\left(\frac{1}{\delta} \mathcal{Q}_{n, b}^{\left(\mu_{n}, v_{n}\right)}\left((t-y)^{2} ; y\right)\right) \omega\left(f^{\prime}, \delta\right) \\
& =\left(\left|f^{\prime}(y)\right|+\omega\left(f^{\prime}, \delta\right)\right) \delta_{n}(y)+\left(\frac{\delta_{n}^{2}(y)}{\delta}\right) \omega\left(f^{\prime}, \delta\right) .
\end{aligned}
$$

Choosing $\delta=\delta_{n}(y)$, we get our desired result.

For $f \in \tilde{C}_{B}[0, \infty)$, the Ditzian-Totik modulus of smoothness [4] of the first order is given by

$$
\omega_{\varphi}(f, \delta)=\sup _{0<h \leq \delta}\left\{\left|f\left(y+\frac{h \varphi(y)}{2}\right)-f\left(y-\frac{h \varphi(y)}{2}\right)\right| ; y \pm \frac{h \varphi(y)}{2} \in[0, \infty)\right\}
$$

and an appropriate Peetre's $K$-functional is defined by

$$
K_{\varphi}(f, \delta)=\inf _{g \in W_{\varphi}[0, \infty)}\left\{\|f-g\|_{\infty}+\delta\left\|\varphi g^{\prime}\right\|_{\infty}\right\}, \quad \delta>0
$$

where $W_{\varphi}[0, \infty):=\left\{g: g \in \mathrm{AC}_{\text {loc }}[0, \infty),\left\|\varphi g^{\prime}\right\|_{\infty}<\infty\right\}$ where $g \in \mathrm{AC}_{\text {loc }}[0, \infty)$ means $g$ is absolutely continuous on every compact subset $[a, b]$ of $[0, \infty)$. It is known from [4] that there exists a constant $M$ such that

$$
M^{-1} \omega_{\varphi}(f, \delta) \leq K_{\varphi}(f, \delta) \leq M \omega_{\varphi}(f, \delta)
$$

Now, we find the order of approximation of the sequence of operators (1.3) by means of Ditzian-Totik modulus of smoothness.

Theorem 3.7 For any $f \in \tilde{C}_{B}[0, \infty)$ and $y \in[0, \infty)$,

$$
\left|\mathcal{Q}_{n, b}^{\left(\mu_{n}, v_{n}\right)}(f ; y)-f(y)\right| \leq M \omega_{\varphi}\left(f, \frac{\delta_{n}(y)}{\sqrt{y}}\right)
$$

Proof Let $\varphi(y)=\sqrt{y}$, then by Taylor's theorem, for any $g \in W_{\varphi}[0, \infty)$, we get

$$
g(t)=g(y)+\int_{y}^{t} g^{\prime}(u) d u=g(y)+\int_{y}^{t} \frac{g^{\prime}(u) \varphi(u)}{\varphi(u)} d u,
$$

therefore,

$$
\begin{aligned}
|g(t)-g(y)| & =\left\|\varphi g^{\prime}\right\|_{\infty}\left|\int_{y}^{t} \frac{1}{\varphi(u) d u}\right| \\
& =2\left\|\varphi g^{\prime}\right\|_{\infty}|\sqrt{t}-\sqrt{y}| \\
& =2\left\|\varphi g^{\prime}\right\|_{\infty} \frac{|t-y|}{\sqrt{t}+\sqrt{y}}
\end{aligned}
$$


which gives

$$
|g(t)-g(y)| \leq 2\left\|\varphi g^{\prime}\right\|_{\infty} \frac{|t-y|}{\sqrt{y}}=2\left\|\varphi g^{\prime}\right\|_{\infty} \frac{|t-y|}{\varphi(y)} .
$$

Using Lemma 2.1 and the above equation, for any $g \in W_{\varphi}[0, \infty)$, we get

$$
\begin{aligned}
\left|\mathcal{Q}_{n, b}^{\left(\mu_{n}, v_{n}\right)}(f ; y)-f(y)\right| & \leq\left|\mathcal{Q}_{n, b}^{\left(\mu_{n}, v_{n}\right)}(f-g ; y)\right|+\left|\mathcal{Q}_{n, b}^{\left(\mu_{n}, v_{n}\right)}(g ; y)-g(y)\right|+|g(y)-f(y)| \\
& \leq 2\|f-g\|_{\infty}+\frac{2\left\|\varphi g^{\prime}\right\|_{\infty}}{\varphi(y)} \mathcal{Q}_{n, b}^{\left(\mu_{n}, v_{n}\right)}(|t-y| ; y) .
\end{aligned}
$$

Applying the Cauchy-Schwarz inequality yields

$$
\begin{aligned}
\left|\mathcal{Q}_{n, b}^{\left(\mu_{n}, v_{n}\right)}(f ; y)-f(y)\right| & \leq 2\|f-g\|_{\infty}+\frac{2\left\|\varphi g^{\prime}\right\|_{\infty}}{\varphi(y)} \sqrt{\mathcal{Q}_{n, b}^{\left(\mu_{n}, v_{n}\right)}\left((t-y)^{2} ; y\right)} \\
& =2\|f-g\|_{\infty}+\frac{2\left\|\varphi g^{\prime}\right\|_{\infty}}{\varphi(y)} \delta_{n}(y) .
\end{aligned}
$$

Taking infimum on the right-hand side over all $g \in W_{\varphi}[0, \infty)$, we get

$$
\left|\mathcal{Q}_{n, b}^{\left(\mu_{n}, v_{n}\right)}(f ; y)-f(y)\right| \leq 2 K_{\varphi}\left(f, \frac{\delta_{n}(y)}{\sqrt{y}}\right)
$$

which leads to the required result with the help of the relation between Peetre's $K$ functional and Ditzian-Totik modulus of smoothness as given by the relation (3.9).

\section{Approximation results in weighted spaces}

Let $v>0$. We denote $C_{v}[0, \infty):=\left\{f \in C[0, \infty):|f(t)| \leq M_{f}\left(1+t^{v}\right), \forall t \geq 0\right\}$ equipped with the norm

$$
\|f\|_{\nu}=\sup _{t \in[0, \infty)} \frac{|f(t)|}{1+t^{\nu}}
$$

Further, let $C_{2}^{*}[0, \infty)$ be the subspace of $C_{2}[0, \infty)$ consisting of functions $f$ such that $\lim _{t \rightarrow \infty} \frac{f(t)}{1+t^{2}}$ exists.

Theorem 4.1 For each $f \in C_{2}^{*}[0, \infty)$ and $r>0$, the following relation holds:

$$
\lim _{n \rightarrow \infty} \sup _{x \in[0, \infty)} \frac{\left|\mathcal{Q}_{n, b}^{\left(\mu_{n}, v_{n}\right)}(f ; y)-f(y)\right|}{\left(1+y^{2}\right)^{1+r}}=0 .
$$

Proof Let $y_{0}>0$ be arbitrary but fixed, then by (4.1), we can write

$$
\begin{aligned}
& \sup _{y \in[0, \infty)} \frac{\left|\mathcal{Q}_{n, b}^{\left(\mu_{n}, \nu_{n}\right)}(f ; y)-f(y)\right|}{\left(1+y^{2}\right)^{1+r}} \\
& \quad \leq \sup _{y \leq y_{0}} \frac{\left|\mathcal{Q}_{n, b}^{\left(\mu_{n}, v_{n}\right)}(f ; y)-f(y)\right|}{\left(1+y^{2}\right)^{1+r}}+\sup _{y>y_{0}} \frac{\left|\mathcal{Q}_{n, b}^{\left(\mu_{n}, \nu_{n}\right)}(f ; y)-f(y)\right|}{\left(1+y^{2}\right)^{1+r}}
\end{aligned}
$$




$$
\leq \sup _{y \leq y_{0}}\left\{\left|\mathcal{Q}_{n, b}^{\left(\mu_{n}, v_{n}\right)}(f ; y)-f(y)\right|\right\}+\sup _{y>y_{0}} \frac{\left|\mathcal{Q}_{n, b}^{\left(\mu_{n}, v_{n}\right)}(f ; y)+f(y)\right|}{\left(1+y^{2}\right)^{1+r}} .
$$

Since $|f(t)| \leq\|f\|_{2}\left(1+y^{2}\right)$, we get

$$
\begin{aligned}
& \sup _{y \in[0, \infty)} \frac{\left|\mathcal{Q}_{n, b}^{\left(\mu_{n}, v_{n}\right)}(f ; y)-f(y)\right|}{\left(1+y^{2}\right)^{1+r}} \\
& \quad \leq\left\|\mathcal{Q}_{n, b}^{\left(\mu_{n}, v_{n}\right)}(f ; y)-f(y)\right\|_{C\left[0, y_{0}\right]}+\|f\|_{2} \sup _{y>y_{0}} \frac{\left|\mathcal{Q}_{n, b}^{\left(\mu_{n}, v_{n}\right)}\left(1+t^{2} ; y\right)\right|}{\left(1+y^{2}\right)^{1+r}}+\sup _{y>y_{0}} \frac{\|f(y)\|}{\left(1+y^{2}\right)^{1+r}} \\
& \quad=I_{1}+I_{2}+I_{3} \quad \text { (say). }
\end{aligned}
$$

By Korovkin's theorem, we can see that the sequence of operators $\left\{\mathcal{Q}_{n, b}^{\left(\mu_{n}, v_{n}\right)}(f ; y)\right\}$ converges uniformly to the function $f$ on every closed interval $[0, a]$ as $n \rightarrow \infty$, (cf. [12, p. 149]). Therefore, for a given $\epsilon>0, \exists n_{1} \in \mathbb{N}$ such that

$$
I_{1}=\left\|\mathcal{Q}_{n, b}^{\left(\mu_{n}, v_{n}\right)}(f ; y)-f(y)\right\|_{C\left[0, y_{0}\right]}<\frac{\epsilon}{3}, \quad \forall n \geq n_{1} .
$$

By using Lemma 2.1, we can find $n_{2} \in \mathbb{N}$ such that

$$
\begin{aligned}
&\left|\mathcal{Q}_{n, b}^{\left(\mu_{n}, v_{n}\right)}\left(1+t^{2} ; y\right)-\left(1+y^{2}\right)\right|<\frac{\epsilon}{3\|f\|_{2}}, \quad \forall n \geq n_{2}, \\
& \quad \text { or } \quad \mathcal{Q}_{n, b}^{\left(\mu_{n}, v_{n}\right)}\left(1+t^{2} ; y\right)<\left(1+y^{2}\right)+\frac{\epsilon}{3\|f\|_{2}}, \quad \forall n \geq n_{2} .
\end{aligned}
$$

Hence

$$
\begin{aligned}
I_{2} & =\|f\|_{2} \sup _{y>y_{0}} \frac{\left|\mathcal{Q}_{n, b}^{\left(\mu_{n}, v_{n}\right)}\left(1+t^{2} ; y\right)\right|}{\left(1+y^{2}\right)^{1+r}} \\
& <\|f\|_{2} \sup _{y>y_{0}} \frac{1}{\left(1+y^{2}\right)^{1+r}}\left(\left(1+y^{2}\right)+\frac{\epsilon}{3\|f\|_{2}}\right) \\
& <\|f\|_{2} \sup _{y>y_{0}}\left(\frac{1}{\left(1+y^{2}\right)^{r}}\right)+\frac{\epsilon}{3} \\
& <\frac{\|f\|_{2}}{\left(1+y_{0}^{2}\right)^{r}}+\frac{\epsilon}{3}, \quad \forall n \geq n_{2} .
\end{aligned}
$$

Now, using (4.1),

$$
I_{3}=\sup _{y>y_{0}} \frac{|f(y)|}{\left(1+y^{2}\right)^{1+r}} \leq \frac{\|f\|_{2}}{\left(1+y_{0}^{2}\right)^{r}} .
$$

Let us denote $n_{0}=\max \left\{n_{1}, n_{2}\right\}$, then by (4.4), (4.5), and (4.6), we get

$$
I_{1}+I_{2}+I_{3}<2 \frac{\|f\|_{2}}{\left(1+y_{0}^{2}\right)^{r}}+\frac{2 \epsilon}{3}, \quad \forall n \geq n_{0} .
$$

Choose $y_{0}$ so large that

$$
2 \frac{\|f\|_{2}}{\left(1+y_{0}^{2}\right)^{r}}<\frac{\epsilon}{3} .
$$


Then, combining (4.3), (4.7), and (4.8), we obtain

$$
\sup _{y \in[0, \infty)} \frac{\left|\mathcal{Q}_{n, b}^{\left(\mu_{n}, v_{n}\right)}(f ; y)-f(y)\right|}{\left(1+y^{2}\right)^{1+r}}<\epsilon, \quad \forall n \geq n_{0} .
$$

Hence, the proof is completed.

Now, we will obtain the rate of convergence of the operators $\mathcal{Q}_{n, b}^{\left(\mu_{n}, v_{n}\right)}(f ; y)$ defined by (1.3) for the functions having derivatives of bounded variation. Let $\operatorname{DBV}[0, \infty)$ be the space of functions in $C_{2}[0, \infty)$, which have the derivative of bounded variation on every finite subinterval of $[0, \infty)$. Here, we show at the point $y$, where $f^{\prime}(y+)$ and $f^{\prime}(y-)$ exist, the operators $\mathcal{Q}_{n, b}^{\left(\mu_{n}, v_{n}\right)}(f ; y)$ converge to the function $f(y)$. A function $f \in \operatorname{DBV}[0, \infty)$ can be represented as

$$
f(y)=\int_{0}^{y} g(t) d t+f(0)
$$

where $g$ denotes a function of bounded variation on every finite subinterval $[0, \infty)$. Many researchers studied in this direction and their work pertaining to this area is described in the papers $[2,11,19]$, etc.

In order to study the order of convergence of the operators $\mathcal{Q}_{n, b}^{\left(\mu_{n}, v_{n}\right)}(f ; y)$ for the functions having a derivative of bounded variation, we rewrite the operator (1.3) as follows:

$$
\mathcal{Q}_{n, b}^{\left(\mu_{n}, v_{n}\right)}(f ; y)=\int_{0}^{\infty} W(t, y) f(t) d t
$$

where $W(t, y)$ is a kernel given by

$$
W(t, y)=v_{n} e^{-1}\left(1-\frac{1}{b}\right)^{(b-1) \mu_{n} y} \sum_{l=0}^{\infty} \frac{C_{l}^{(b)}\left(-(b-1) \mu_{n} y\right)}{l !} \chi_{I}(t)
$$

$\chi_{I}(t)$ being the characteristic function of $I=\left[\frac{l}{v_{n}}, \frac{l+1}{v_{n}}\right]$.

Lemma 4.2 Let for all $x>0$ and sufficiently large $n$,

(1) $\lambda_{\mu_{n}, v_{n}}(t, y)=\int_{0}^{t} W(u, y) d u \leq \frac{\xi_{\mu_{n}, v_{n}}^{2}(x)}{(y-t)^{2}}, 0 \leq t<y$,

(2) $1-\lambda_{\mu_{n}, v_{n}}(t, y)=\int_{t}^{\infty} W(u, y) d u \leq \frac{\xi_{\mu_{n}, v_{n}}^{2}(y)}{(t-y)^{2}}, y \leq t<\infty$.

Proof Using Lemma 2.1 and the definition of the kernel, we get

$$
\begin{aligned}
\lambda_{\mu_{n}, v_{n}}(t, y) & =\int_{0}^{t} W(u, y) d u \\
& \leq \int_{0}^{t}\left(\frac{y-u}{y-t}\right)^{2} W(u, y) d u \\
& \leq \frac{1}{(y-t)^{2}} \int_{0}^{t}(u-y)^{2} W(u, y) d u .
\end{aligned}
$$


Hence, we have

$$
\begin{aligned}
\lambda_{\mu_{n}, v_{n}}(t, y) & \leq \frac{1}{(y-t)^{2}} \mathcal{Q}_{n, b}^{\left(\mu_{n}, v_{n}\right)}\left((u-y)^{2} ; y, b\right) \\
& \leq \frac{1}{(y-t)^{2}} \xi_{\mu_{n}, v_{n}}^{2}(y) .
\end{aligned}
$$

In the same fashion, we can prove the other inequality, therefore, we omit the details.

Let $\bigvee_{a}^{b} f$ be the total variation of $f$ on $[a, b]$, i.e.,

$$
\bigvee_{a}^{b} f=V(f ;[a, b])=\sup _{P \in \mathbb{P}}\left(\sum_{i=1}^{n}\left|f\left(y_{i}\right)-f\left(y_{i-1}\right)\right|\right),
$$

where $\mathbb{P}$ is the set of all partitions $P=\left\{a=y_{0}, y_{1}, \ldots, y_{n}=b\right\}$ of $[a, b]$, whick also has the property

$$
\bigvee_{a}^{b} f=\bigvee_{a}^{c} f+\bigvee_{c}^{b} f
$$

Let

$$
f_{y}^{\prime}(t)= \begin{cases}f^{\prime}(t)-f^{\prime}(y-), & 0 \leq t<y, \\ 0, & t=y, \\ f^{\prime}(t)-f^{\prime}(y+), & y<t<\infty\end{cases}
$$

Theorem 4.3 Let $f \in \mathrm{DBV}[0, \infty), y>0$, and $n$ be sufficiently large, then we get

$$
\begin{aligned}
\left|\mathcal{Q}_{n, b}^{\left(\mu_{n}, v_{n}\right)}(f ; y)-f(y)\right| & \\
\leq & \left|\frac{1}{2}\left(f^{\prime}(y+)+f^{\prime}(y-)\right)\right|\left|\psi_{\mu_{n}, v_{n}}(y)\right|+\frac{\xi_{\mu_{n}, v_{n}}^{2}(y)}{y} \sum_{k=1}^{[\sqrt{n}]}\left(\bigvee_{y-y / k}^{y+y / k} f_{y}^{\prime}\right)+\frac{y}{\sqrt{n}}\left(\bigvee_{y-y / k}^{y+y / k} f_{y}^{\prime}\right) \\
& +\frac{\xi_{\mu_{n}, v_{n}}^{2}(y)}{y^{2}}\left|f(2 y)-f(y)-y f^{\prime}(y+)\right|+\left(\frac{M_{f}+|f(y)|}{y^{2}}+4 M_{f}\right) \xi_{\mu_{n}, v_{n}}^{2}(y) \\
& +\left|f^{\prime}(y+)\right|\left|\psi_{\mu_{n}, v_{n}}(y)\right|+\left|\frac{1}{2}\left(f^{\prime}(y+)-f^{\prime}(y-)\right)\right| \xi_{\mu_{n}, v_{n}}(y) .
\end{aligned}
$$

Proof By (4.11), we obtain

$$
\begin{aligned}
f^{\prime}(t)= & \frac{1}{2}\left(f^{\prime}(y+)+f^{\prime}(y-)\right)+f_{y}^{\prime}(t)+\frac{1}{2}\left(f^{\prime}(y+)-f^{\prime}(y-)\right) \operatorname{sgn}(t-y) \\
& +\delta_{y}(t)\left(f^{\prime}(t)-\frac{1}{2}\left(f^{\prime}(y+)+f^{\prime}(y-)\right)\right)
\end{aligned}
$$

where

$$
\delta_{y}(t)= \begin{cases}1, & t=y, \\ 0, & t \neq y .\end{cases}
$$


Now using Lemma 2.1, equations (4.9) and (4.12), we get

$$
\begin{aligned}
\mathcal{Q}_{n, b}^{\left(\mu_{n}, v_{n}\right)}(f ; y)-f(y) \\
=\int_{0}^{\infty}(f(t)-f(y)) W(t, y) d t \\
=\int_{0}^{\infty}\left(\int_{y}^{t} f^{\prime}(u) d u\right) W(t, y) d t \\
=\int_{0}^{\infty}\left[\int _ { y } ^ { t } \left\{\frac{1}{2}\left(f^{\prime}(y+)+f^{\prime}(y-)\right)+f_{y}^{\prime}(u)+\frac{1}{2}\left(f^{\prime}(y+)-f^{\prime}(y-)\right) \operatorname{sgn}(u-y)\right.\right. \\
\left.\left.\quad+\delta_{y}(u)\left(f^{\prime}(u)-\frac{1}{2}\left(f^{\prime}(y+)+f^{\prime}(y-)\right)\right)\right\} d u\right] W(t, y) d t .
\end{aligned}
$$

Since $\int_{y}^{t} \delta_{y}(u) d u=0$, we have

$$
\begin{aligned}
\mathcal{Q}_{n, b}^{\left(\mu_{n}, v_{n}\right)}(f ; y)-f(y) & \\
= & \frac{1}{2}\left(f^{\prime}(y+)+f^{\prime}(y-)\right) \int_{0}^{\infty}(t-y) W(t, y) d t+\int_{0}^{\infty}\left(\int_{y}^{t} f_{y}^{\prime}(u) d u\right) W(t, y) d t \\
& \quad+\frac{1}{2}\left(f^{\prime}(y+)-f^{\prime}(y-)\right) \int_{0}^{\infty}|t-y| W(t, y) d t .
\end{aligned}
$$

Now, we break the second term on the right-hand side of the above equation as follows:

$$
\begin{aligned}
& \int_{0}^{\infty}\left(\int_{y}^{t} f_{y}^{\prime}(u) d u\right) W(t, y) d t \\
& \quad=-\int_{0}^{y}\left(\int_{t}^{y} f_{y}^{\prime}(u) d u\right) W(t, y) d t+\int_{y}^{\infty}\left(\int_{y}^{t} f_{y}^{\prime}(u) d u\right) W(t, y) d t \\
& =-I_{1}+I_{2}
\end{aligned}
$$

where

$$
\begin{aligned}
& I_{1}=\int_{0}^{y}\left(\int_{t}^{y} f_{y}^{\prime}(u) d u\right) W(t, y) d t, \\
& I_{2}=\int_{y}^{\infty}\left(\int_{y}^{t} f_{y}^{\prime}(u) d u\right) W(t, y) d t .
\end{aligned}
$$

Taking the absolute value on both sides of (4.13), we have

$$
\begin{aligned}
& \left|\mathcal{Q}_{n, b}^{\left(\mu_{n}, v_{n}\right)}(f ; y)-f(y)\right| \\
& \leq\left|\frac{1}{2}\left(f^{\prime}(y+)+f^{\prime}(y-)\right)\right|\left|\mathcal{Q}_{n, b}^{\left(\mu_{n}, v_{n}\right)}(t-y ; y)\right|+\left|I_{1}\right|+\left|I_{2}\right| \\
& \quad+\left|\frac{1}{2}\left(f^{\prime}(y+)-f^{\prime}(y-)\right)\right| \mathcal{Q}_{n, b}^{\left(\mu_{n}, v_{n}\right)}(|t-y| ; y) .
\end{aligned}
$$


After applying the Cauchy-Schwarz inequality, we obtain

$$
\begin{aligned}
& \left|\mathcal{Q}_{n, b}^{\left(\mu_{n}, v_{n}\right)}(f ; y)-f(y)\right| \\
& \leq\left|\frac{1}{2}\left(f^{\prime}(y+)+f^{\prime}(y-)\right)\right|\left|\psi_{\mu_{n}, v_{n}}(y)\right|+\left|I_{1}\right|+\left|I_{2}\right| \\
& \quad+\left|\frac{1}{2}\left(f^{\prime}(y+)-f^{\prime}(y-)\right)\right| \sqrt{\mathcal{Q}_{n, b}^{\left(\mu_{n}, v_{n}\right)}\left((t-y)^{2} ; y\right)} \\
& =\left|\frac{1}{2}\left(f^{\prime}(y+)+f^{\prime}(y-)\right)\right|\left|\psi_{\mu_{n}, v_{n}}(y)\right|+\left|I_{1}\right|+\left|I_{2}\right|+\left|\frac{1}{2}\left(f^{\prime}(y+)-f^{\prime}(y-)\right)\right| \xi_{\mu_{n}, v_{n}}(y) .
\end{aligned}
$$

Now applying Lemma 4.2 and integration by parts, $I_{1}$ can be written as

$$
\begin{aligned}
I_{1} & =\int_{0}^{y}\left(\int_{t}^{y} f_{y}^{\prime}(u) d u\right) W(t, y) d t \\
& =\int_{0}^{y}\left(\int_{t}^{y} f_{y}^{\prime}(u) d u\right) \frac{\partial}{\partial t} \lambda_{\mu_{n}, v_{n}}(t, y) d t \\
& =\int_{0}^{y} f_{y}^{\prime}(t) \lambda_{\mu_{n}, v_{n}}(t, y) d t .
\end{aligned}
$$

On taking the absolute value of $I_{1}$, we have

$$
\begin{aligned}
\left|I_{1}\right| & =\int_{0}^{y}\left|f_{y}^{\prime}(t)\right| \lambda_{\mu_{n}, v_{n}}(t, y) d t \\
& \leq \int_{0}^{y-y / \sqrt{n}}\left|f_{y}^{\prime}(t)\right| \lambda_{\mu_{n}, v_{n}}(t, y) d t+\int_{y-y / \sqrt{n}}^{y}\left|f_{y}^{\prime}(t)\right| \lambda_{\mu_{n}, v_{n}}(t, y) d t \\
& =K_{1}+K_{2}, \quad \text { say. }
\end{aligned}
$$

Since $f_{y}^{\prime}(y)=0$, by (4.11), we have

$$
K_{1}=\int_{0}^{y-y / \sqrt{n}}\left|f_{y}^{\prime}(t)-f_{y}^{\prime}(y)\right| \lambda_{\mu_{n}, v_{n}}(t, y) d t .
$$

Now, using Lemma 4.2,

$$
K_{1} \leq \xi_{\mu_{n}, v_{n}}^{2}(y) \int_{0}^{y-y / \sqrt{n}}\left|f_{y}^{\prime}(t)-f_{y}^{\prime}(y)\right| \frac{d t}{(y-t)^{2}} .
$$

By the definition of total variation (4.10) and taking $t=y-y / u$, we obtain

$$
\begin{aligned}
K_{1} & \leq \xi_{\mu_{n}, v_{n}}^{2}(y) \int_{0}^{y-y / \sqrt{n}}\left(\bigvee_{t}^{y} f_{y}^{\prime}\right) \frac{d t}{(y-t)^{2}} \\
& =\xi_{\mu_{n}, v_{n}}^{2}(y) \int_{1}^{\sqrt{n}}\left(\bigvee_{y-y / u}^{y} f_{y}^{\prime}\right) \frac{d u}{y}
\end{aligned}
$$


Now, after breaking the integral into a sum, we have

$$
\begin{aligned}
K_{1} & \leq \frac{\xi_{\mu_{n}, v_{n}}^{2}(y)}{y} \sum_{k=1}^{[\sqrt{n}]} \int_{k}^{k+1}\left(\bigvee_{y-y / u}^{y} f_{y}^{\prime}\right) d u \\
& \leq \frac{\xi_{\mu_{n}, v_{n}}^{2}(y)}{y} \sum_{k=1}^{[\sqrt{n}]}\left(\bigvee_{y-y / k}^{y} f_{y}^{\prime}\right)\left(\int_{k}^{k+1} d u\right) \\
& =\frac{\xi_{\mu_{n}, v_{n}}^{2}(y)}{y} \sum_{k=1}^{[\sqrt{n}]}\left(\bigvee_{y-y / k}^{y} f_{y}^{\prime}\right) .
\end{aligned}
$$

Since by Lemma $4.2, \lambda_{\mu_{n}, v_{n}}(t, y) \leq 1$ and using (4.11), we get

$$
\begin{aligned}
K_{2} & =\int_{y-y / n}^{y}\left(\bigvee_{t}^{y} f_{y}^{\prime}\right) d t \\
& \leq\left(\bigvee_{y-y / n}^{y} f_{y}^{\prime}\right) \int_{y-y / n}^{y} d t \\
& =\frac{y}{\sqrt{n}}\left(\bigvee_{y-y / n}^{y} f_{y}^{\prime}\right) .
\end{aligned}
$$

Thus, we get

$$
\left|I_{1}\right| \leq \frac{\xi_{\mu_{n}, v_{n}}^{2}(y)}{y} \sum_{k=1}^{[\sqrt{n}]}\left(\bigvee_{y-y / k}^{y} f_{y}^{\prime}\right)+\frac{y}{\sqrt{n}}\left(\bigvee_{y-y / n}^{y} f_{y}^{\prime}\right)
$$

Using Lemma 4.2, we can write

$$
\begin{aligned}
\left|I_{2}\right| & =\left|\int_{y}^{\infty}\left(\int_{y}^{t} f_{y}^{\prime}(u) d u\right) W(t, y) d t\right| \\
& \leq\left|\int_{y}^{2 y}\left(\int_{y}^{t} f_{y}^{\prime}(u) d u\right) \frac{\partial}{\partial t}\left(1-\lambda_{\mu_{n}, v_{n}}(t, y)\right) d t\right|+\left|\int_{2 y}^{\infty}\left(\int_{y}^{t} f_{y}^{\prime}(u) d u\right) W(t, y) d t\right| .
\end{aligned}
$$

Now, applying integration by parts and (4.11), we get

$$
\begin{aligned}
\left|I_{2}\right|= & \left|\int_{y}^{2 y} f_{y}^{\prime}(u) d u\left(1-\lambda_{\mu_{n}, v_{n}}(2 y, y)\right)-\int_{y}^{2 y} f_{y}^{\prime}(t)\left(1-\lambda_{\mu_{n}, v_{n}}(t, y)\right) d t\right| \\
& +\left|\int_{2 y}^{\infty}\left(\int_{y}^{t}\left(f^{\prime}(u)-f^{\prime}(y+)\right) d u\right) W(t, y) d t\right| \\
\leq & \left|\int_{y}^{2 y} f_{y}^{\prime}(u) d u\right| \cdot \frac{\xi_{\mu_{n}, v_{n}}^{2}(y)}{y^{2}}+\int_{y}^{2 y}\left|f_{y}^{\prime}(t)\right|\left(1-\lambda_{\mu_{n}, v_{n}}\right)(t, y) d t \\
& +\left|\int_{2 y}^{\infty}(f(t)-f(y)) W(t, y) d t\right|+\left|f^{\prime}(y+)\right|\left|\int_{2 y}^{\infty}(t-y) W(t, y) d t\right| \\
= & P_{1}+P_{2}+P_{3}+P_{4}, \quad \text { say. }
\end{aligned}
$$


Now, by (4.11), we get

$$
\begin{aligned}
P_{1} & =\frac{\xi_{\mu_{n}, v_{n}}^{2}(y)}{y^{2}}\left|\int_{y}^{2 y} f_{y}^{\prime}(u) d u\right| \\
& =\frac{\xi_{\mu_{n}, v_{n}}^{2}(y)}{y^{2}}\left|\int_{y}^{2 y}\left(f^{\prime}(u)-f^{\prime}(y+)\right) d u\right| \\
& \leq \frac{\xi_{\mu_{n}, v_{n}}^{2}(y)}{y^{2}}\left|f(2 y)-f(y)-y f^{\prime}(y+)\right|
\end{aligned}
$$

and

$$
\begin{aligned}
P_{2}= & \int_{y}^{2 y}\left|f_{y}^{\prime}(t)\right| \cdot\left(1-\lambda_{\mu_{n}, v_{n}}(t, y)\right) d t \\
= & \int_{y}^{y+y / \sqrt{n}}\left|f_{y}^{\prime}(t)\right| \cdot\left(1-\lambda_{\mu_{n}, v_{n}}(t, y)\right) d t \\
& +\int_{y+y / \sqrt{n}}^{2 y}\left|f_{y}^{\prime}(t)\right| \cdot\left(1-\lambda_{\mu_{n}, v_{n}}(t, y)\right) d t \\
= & J_{1}+J_{2}, \quad \text { say. }
\end{aligned}
$$

Using Lemma $4.2,1-\lambda_{\mu_{n}, v_{n}}(t, y) \leq 1$ and (4.11), we get

$$
\begin{aligned}
J_{1} & =\int_{y}^{y+y / \sqrt{n}}\left|f_{y}^{\prime}(t)\right| \cdot\left(1-\lambda_{\mu_{n}, v_{n}}(t, y)\right) d t \\
& \leq \int_{y}^{y+y / \sqrt{n}}\left|f_{y}^{\prime}(t)-f_{y}^{\prime}(y)\right| d t \\
& \leq \int_{y}^{y+y / \sqrt{n}}\left(\bigvee_{y}^{t} f_{y}^{\prime}\right) d t \\
& \left.\leq \bigvee_{y}^{y+y / \sqrt{n}} f_{y}^{\prime}\right) \int_{y}^{y+y / \sqrt{n}} d t \\
& \leq \frac{y}{\sqrt{n}}\left(\begin{array}{c}
y+y / \sqrt{n} \\
y
\end{array} f_{y}^{\prime}\right.
\end{aligned}
$$

Now, again with the help of Lemma 4.2 and (4.11), we obtain

$$
\begin{aligned}
J_{2} & =\int_{y+y / \sqrt{n}}^{2 y}\left|f_{y}^{\prime}(t)\right|\left(1-\lambda_{\mu_{n}, v_{n}}(t, y)\right) d t \\
& \leq \xi_{\mu_{n}, v_{n}}^{2}(y) \int_{y+y / \sqrt{n}}^{2 y}\left|f_{y}^{\prime}(t)-f_{y}^{\prime}(y)\right| \frac{d t}{(t-y)^{2}} .
\end{aligned}
$$

By using (4.10) and $t=y+y / u$, we get

$$
J_{2} \leq \xi_{\mu_{n}, v_{n}}^{2}(y) \int_{y+y / \sqrt{n}}^{2 y}\left(\bigvee_{y}^{t} f_{y}^{\prime}\right) \frac{d t}{(t-y)^{2}}
$$




$$
\begin{aligned}
& =\xi_{\mu_{n}, v_{n}}^{2}(y) \int_{1}^{\sqrt{n}}\left(\bigvee_{y}^{y+y / u} f_{y}^{\prime}\right) \frac{d u}{y} \\
& \leq \frac{\xi_{\mu_{n}, v_{n}}^{2}(y)}{y} \sum_{k=1}^{[\sqrt{n}]} \int_{k}^{k+1}\left(\bigvee_{y}^{y+y / u} f_{y}^{\prime}\right) d u \\
& \leq \frac{\xi_{\mu_{n}, v_{n}}^{2}(y)}{y} \sum_{k=1}^{[\sqrt{n}]}\left(\bigvee_{y}^{y+y / k} f_{y}^{\prime}\right)\left(\int_{k}^{k+1} d u\right) \\
& =\frac{\xi_{\mu_{n}, v_{n}}^{2}(y)}{y} \sum_{k=1}^{[\sqrt{n}]}\left(\bigvee_{y}^{y+y / k} f_{y}^{\prime}\right) .
\end{aligned}
$$

Hence, we derive

$$
P_{2} \leq \frac{y}{\sqrt{n}}\left(\bigvee_{y}^{y+y / \sqrt{n}} f_{y}^{\prime}\right)+\frac{\xi_{\mu_{n}, v_{n}}^{2}(y)}{y} \sum_{k=1}^{[\sqrt{n}]}\left(\bigvee_{y}^{y+y / k} f_{y}^{\prime}\right)
$$

Now, we estimate $P_{3}$. As $t \geq 2 y$, then using $2(t-y) \geq t$ and $t-y \geq y$, we get

$$
\begin{aligned}
P_{3} & =\left|\int_{2 y}^{\infty}(f(t)-f(y)) W(t, y) d t\right| \\
& \leq \int_{2 y}^{\infty}|f(t)| W(t, y) d t+\int_{2 y}^{\infty}|f(y)| W(t, y) d t \\
& \leq M_{f} \int_{2 y}^{\infty}\left(1+t^{2}\right) W(t, y) d t+|f(y)| \int_{2 y}^{\infty} W(t, y) d t \\
& =\left(M_{f}+|f(y)|\right) \int_{2 y}^{\infty} W(t, y) d t+M_{f} \int_{2 y}^{\infty} t^{2} W(t, y) d t \\
& \leq\left(M_{f}+|f(y)|\right) \int_{2 y}^{\infty} \frac{(t-y)^{2}}{y^{2}} W(t, y) d t+M_{f} \int_{2 y}^{\infty} 4(t-y)^{2} W(t, y) d t \\
& \leq\left(\frac{M_{f}+|f(y)|}{y^{2}}+4 M_{f}\right) \int_{2 y}^{\infty}(t-y)^{2} W(t, y) d t \\
& \leq\left(\frac{M_{f}+|f(y)|}{y^{2}}+4 M_{f}\right) \mathcal{Q}_{n, b}^{\left(\mu_{n}, v_{n}\right)}\left((t-y)^{2} ; y\right) \\
& =\left(\frac{M_{f}+|f(y)|}{y^{2}}+4 M_{f}\right) \xi_{\mu_{n}, v_{n}}^{2}(y) .
\end{aligned}
$$

We can compute $P_{4}$ as follows:

$$
\begin{aligned}
P_{4} & =\left|f^{\prime}(y+)\right|\left|\int_{2 y}^{\infty}(t-y) W(t, y) d t\right| \\
& \leq\left|f^{\prime}(y+)\right|\left|\int_{0}^{\infty}(t-y) W(t, y) d t\right| \\
& =\left|f^{\prime}(y+)\right|\left|\mathcal{Q}_{n, b}^{\left(\mu_{n}, v_{n}\right)}(t-y ; y)\right| \\
& =\left|f^{\prime}(y+)\right|\left|\psi_{\mu_{n}, v_{n}}(y)\right| .
\end{aligned}
$$


Hence, we get

$$
\begin{aligned}
\left|I_{2}\right| \leq & \frac{\xi_{\mu_{n}, v_{n}}^{2}(y)}{y^{2}}\left|f(2 y)-f(y)-y f^{\prime}(y+)\right| \\
& +\frac{y}{\sqrt{n}}\left(\bigvee_{y}^{y+y / \sqrt{n}} f_{y}^{\prime}\right)+\frac{\xi_{\mu_{n}, v_{n}}^{2}(y)}{y} \sum_{k=1}^{[\sqrt{n}]}\left(\bigvee_{y}^{y+y / k} f_{y}^{\prime}\right) \\
& +\left(\frac{M_{f}+|f(y)|}{y^{2}}+4 M_{f}\right) \xi_{\mu_{n}, v_{n}}^{2}(y)+\left|f^{\prime}(y+)\right|\left|\psi_{\mu_{n}, v_{n}}(y)\right| .
\end{aligned}
$$

Now, from (4.14)-(4.16), we obtain

$$
\begin{aligned}
\left|\mathcal{Q}_{n, b}^{\left(\mu_{n}, v_{n}\right)}(f ; y)-f(y)\right| \leq & \frac{1}{2}\left(f^{\prime}(y+)+f^{\prime}(y-)\right)|| \psi_{\mu_{n}, v_{n}}(y)|+| I_{1}|+| I_{2} \mid \\
& +\left|\frac{1}{2}\left(f^{\prime}(y+)-f^{\prime}(y-)\right)\right| \xi_{\mu_{n}, v_{n}}(y) \\
\leq & \left|\frac{1}{2}\left(f^{\prime}(y+)+f^{\prime}(y-)\right)\right|\left|\psi_{\mu_{n}, v_{n}}(y)\right| \\
& +\frac{\xi_{\mu_{n}, v_{n}}^{2}(y)}{y} \sum_{k=1}^{[\sqrt{n}]}\left(\bigvee_{y-y / k}^{y} f_{y}^{\prime}\right)+\frac{y}{\sqrt{n}}\left(\bigvee_{y-y / \sqrt{n}}^{y} f_{y}^{\prime}\right) \\
& +\frac{\xi_{\mu_{n}, v_{n}}^{2}(y)}{y^{2}}\left|f(2 y)-f(y)-y f^{\prime}(y+)\right| \\
& +\frac{y}{\sqrt{n}}\left(\bigvee_{y}^{y+y / \sqrt{n}} f_{y}^{\prime}\right)+\frac{\xi_{\mu_{n}, v_{n}}^{2}(y)}{y} \sum_{k=1}^{[\sqrt{n}]}\left(\bigvee_{y}^{y+y / k} f_{y}^{\prime}\right) \\
& +\left(\frac{M_{f}+|f(y)|}{y^{2}}+4 M_{f}\right) \xi_{\mu_{n}, v_{n}}^{2}(y)+\left|f^{\prime}(y+)\right|\left|\psi_{\mu_{n}, v_{n}}(y)\right| \\
& +\left|\frac{1}{2}\left(f^{\prime}(y+)-f^{\prime}(y-)\right)\right| \xi_{\mu_{n}, v_{n}}(y),
\end{aligned}
$$

which gives the desired result.

\section{Graphical examples}

Example 5.1 Let us take $f(x)=5 x^{4}-11 x^{3}+2 x^{2}$. The convergence of the sequence of operators defined by Eq. (1.3) when $\mu_{n}=v_{n}=n$ towards the function $f(x)$ (cyan) is shown for $n=10,50,100$, respectively, in Figs. $1-3$ taking $b=2$ (blue), $b=6$ (black), and $b=15$ (red). Figures 4-6 illustrate the convergence of the sequence of operators defined by Eq. (1.3) taking $\mu_{n}=n+\sqrt{n+1}, v_{n}=n+12$ towards the function $f(x)$ (cyan) for $n=10,50,100$, keeping the value of $b$ the same.

Also, a direct comparison between the convergence of the old operator applied to $f$ (when $\mu_{n}=v_{n}=n$ discussed in [9]) (blue) and the new operator (red) defined in Eq. (1.3) towards $f(x)$ (cyan) is shown in Figs. 7-9, respectively, for $n=10,50,100$, and $b=10$. It is clear that the new operator exhibits faster convergence towards the limit than the old operator. Also, the new operator is giving flexibility in choosing parameters in the form of the sequences $\mu_{n}$ and $v_{n}$. 
Figure 1 Convergence of the operators when $\mu_{n}$ $=v_{n}=n$ and $n=10$

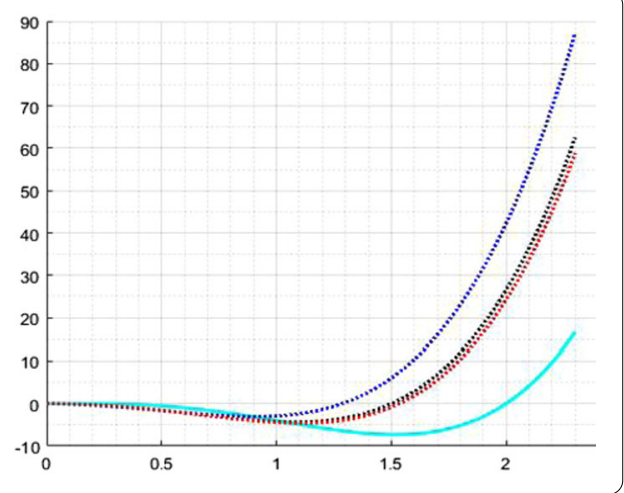

Figure 2 Convergence of the operators when $\mu_{n}$ $=v_{n}=n$ and $n=50$

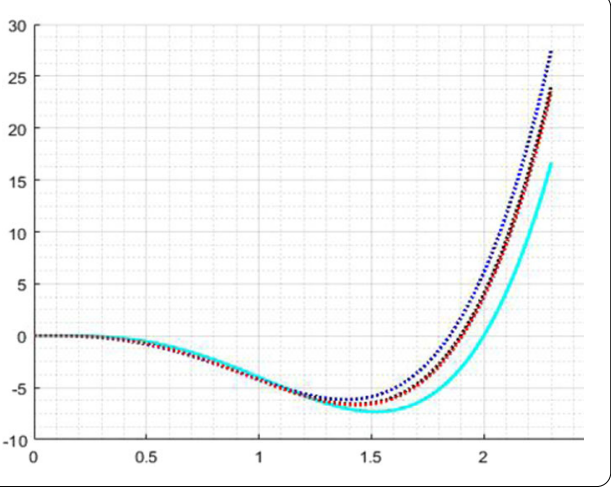

Figure 3 Convergence of the operators when $\mu_{n}$ $=v_{n}=n$ and $n=100$

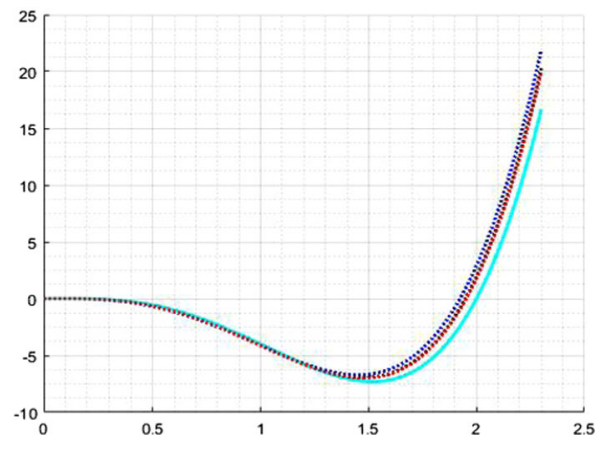

Figure 4 Convergence of the operators when $\mu_{n}$ $=n+\sqrt{n+1}, v_{n}=n+12$ and $n=10$

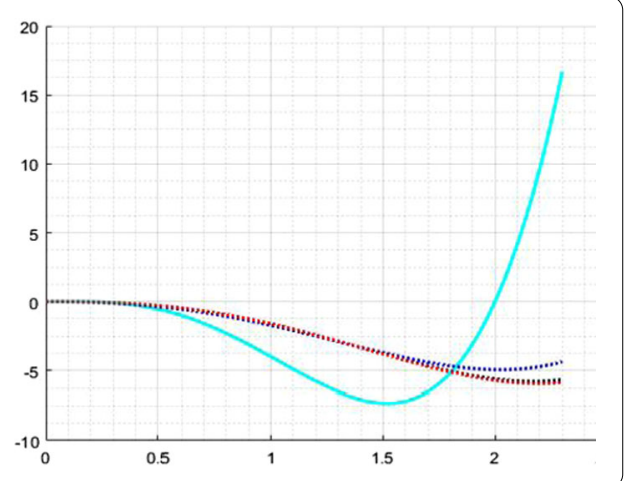


Figure 5 Convergence of the operators when $\mu_{n}$ $=n+\sqrt{n+1}, v_{n}=n+12$ and $n=50$

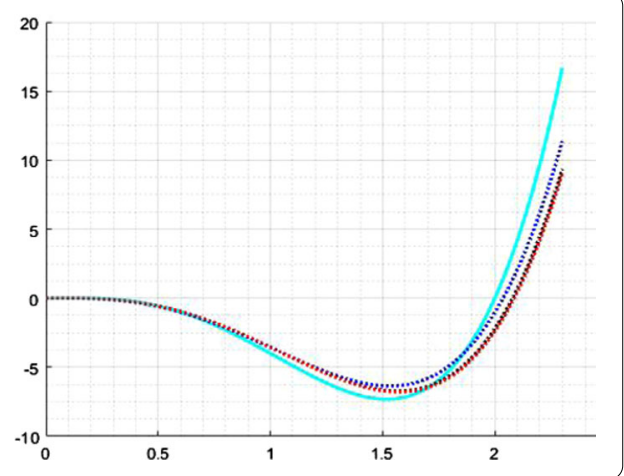

Figure 6 Convergence of the operators when $\mu_{n}$ $=n+\sqrt{n+1}, v_{n}=n+12$ and $n=100$

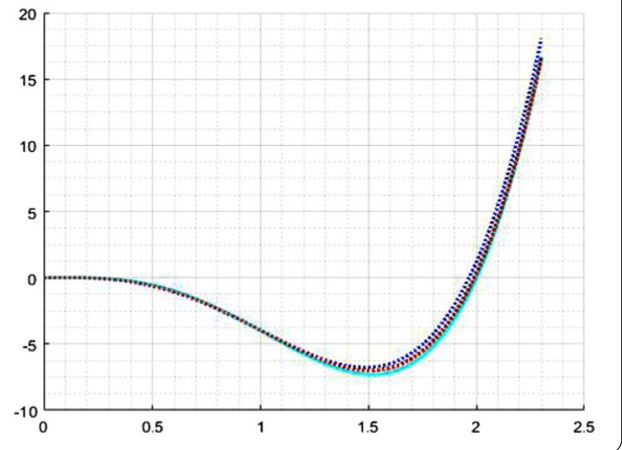

Figure 7 Comparison between the operators when $\mu_{n}=v_{n}=n, b=10$ and $n=10$

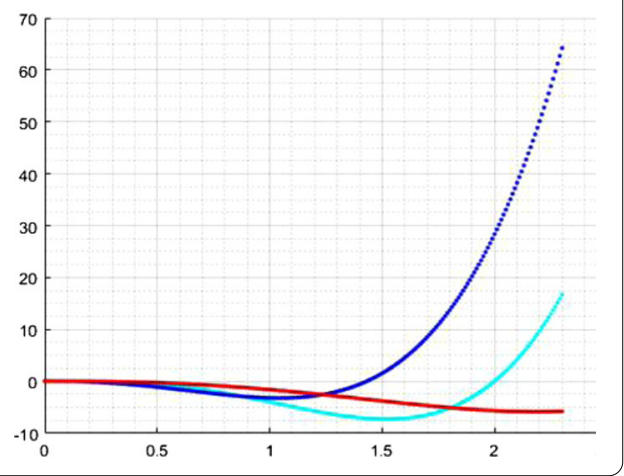

Figure 8 Comparison between the operators when $\mu_{n}=v_{n}=n, b=10$ and $n=50$

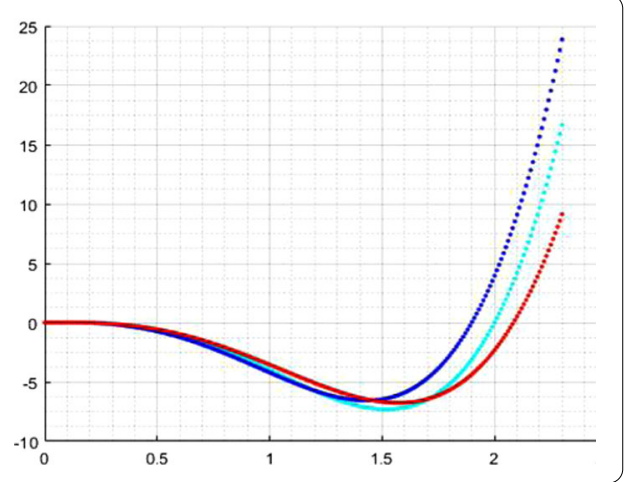


Figure 9 Comparison between the operators when $\mu_{n}=v_{n}=n, b=10$ and $n=100$

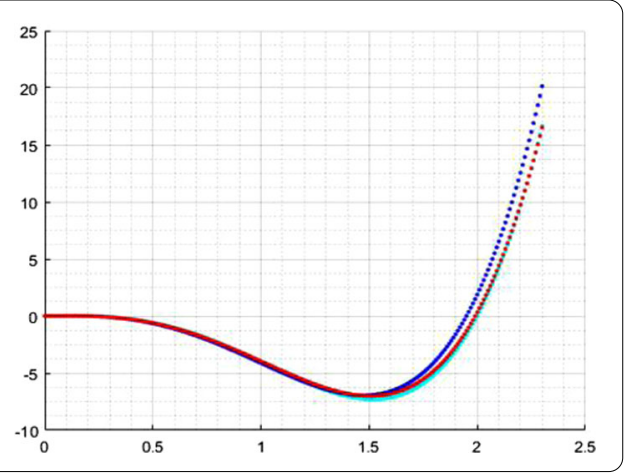

\section{Conclusions}

We have modified the sequence of operators discussed in [9] and developed many approximation properties such as direct theorems, rate of convergence in weighted spaces, and approximation for functions of bounded variation. Moreover, we have also shown the convergence of old and modified new operators graphically.

\section{Acknowledgements}

The author (K.J. Ansari) extends his appreciation to the Deanship of Scientific Research at King Khalid University for funding this work through research groups program under grant number G.R.P-93-41.

Funding

Not applicable.

Availability of data and materials

Not applicable.

Competing interests

The authors declare that they have no competing interests.

\section{Authors' contributions}

The authors contributed equally and significantly in writing this paper. All authors read and approved the final manuscript.

\section{Author details}

${ }^{1}$ Department of Mathematics, College of Science, King Khalid University, Saudi Arabia, Saudi Arabia. ${ }^{2}$ Department of Medical Research, China Medical University Hospital, China Medical University (Taiwan), Taichung, Taiwan. ${ }^{3}$ Department of Mathematics, Aligarh Muslim University, Aligarh, India. ${ }^{4}$ Department of Computer Science and Information Engineering, Asia University, Taichung, Taiwan. ${ }^{5}$ Department of IT, Ibra College of Technology, Ibra, Sultanate of Oman. ${ }^{6}$ Department of Computer Science, College of Computer Science, King Khalid University, Gregor, Abha, Saudi Arabia.

\section{Publisher's Note}

Springer Nature remains neutral with regard to jurisdictional claims in published maps and institutional affiliations.

Received: 12 February 2020 Accepted: 16 April 2020 Published online: 01 May 2020

\section{References}

1. Agrawal, P.N., İspir, N.: Degree of approximation for bivariate Chlodowsky-Szász-Charlier type operators. Results Math. 69, 369-385 (2016)

2. Ansari, K.J, Mursaleen, M., Al-Abeid, A.H.: Approximation by Chlodowsky variant of Szász operators involving Sheffer polynomials. Adv. Oper. Theory 4(2), 321-341 (2019)

3. Atakut, Ç., Büyükyazici, I.: Approximation by Kantorovich-Szász type operators based on Brenke type polynomials. Numer. Funct. Anal. Optim. 37(12), 1488-1502 (2016)

4. Ditzian, Z., Totik, V.: Moduli of Smoothness. Springer Series in Computational Mathematics, vol. 8. Springer, New York (1987)

5. Ismail, M.E.H.: Classical and Quantum Orthogonal Polynomials in One Variable. Cambridge University Press, Cambridge (2005)

6. Kajla, A.: Statistical approximation of Szász type operators based on Charlier polynomials. Kyungpook Math. J. 59, 679-688 (2019)

7. Kajla, A., Agrawal, P.N.: Szász-Durrmeyer type operators based on Charlier polynomials. Appl. Math. Comput. 268, 1001-1014(2015) 
8. Kajla, A., Agrawal, P.N.: Approximation properties of Szász type operators based on Charlier polynomials. Turk. J. Math. 39, 990-1003 (2015)

9. Kajla, A., Agrawal, P.N.: Szász-Kantorovich type operators based on Charlier polynomials. Kyungpook Math. J. 56, 877-897 (2016)

10. Kantorovich, L.V.: Sur certains développements suivant les polynômes la forme de S. Bernstein, I, II, C. R. Acad. URSS, 563-568, 595-600 (1930)

11. Karsli, $\mathrm{H}$.: Rate of convergence of new gamma type operators for functions with derivatives of bounded variation. Math. Comput. Model. 45(5-6), 617-624 (2007)

12. Korovkin, P.P.: On convergence of linear positive operators in the space of continuous functions. Dokl. Akad. Nauk SSSR 90, 961-964 (1953) (Russian)

13. Lenze, B.: Bernstein-Baskakov-Kantorovic operators and Lipschitz-type maximal functions. In: Approximation Theory, Kecskemet, 1990, Colloq. Math. Soc. Janos Bolyai, vol. 58, pp. 469-496, North-Holland, Amsterdam (1991)

14. Mursaleen, M., Al-Abeid, A.H., Ansari, K.J.: On approximation properties of Baskakov-Schurer-Szász-Stancu operators based on q-integers. Filomat 32(4), 1359-1378 (2018)

15. Mursaleen, M., Alotaibi, A., Ansari, K.J.: On a Kantorovich variant of (p,q)-Szász-Mirakjan operators. J. Funct. Spaces 2016, Article ID 1035253 (2016)

16. Mursaleen, M. Ansari, K.J.: On Chlodowsky variant of Szász operators by Brenke type polynomials. Appl. Math. Comput. 271, 991-1003 (2015)

17. Mursaleen, M., Rahman, S., Ansari, K.J.: Approximation by generalized Stancu type integral operators involving Sheffer polynomials. Carpath. J. Math. 34(2), 215-228 (2018)

18. Özarslan, M.A., Aktuğlu, H.: Local approximation properties for certain King type operators. Filomat 27(1), 173-181 (2013)

19. Ozarslan, M.A., Duman, O., Kaanoğlu, C.: Rates of convergence of certain King-type operators for functions with derivative of bounded variation. Math. Comput. Model. 52(1-2), 334-345 (2010)

20. Szász, O.: Generalization of S. Bernstein's polynomials to the infinite interval. J. Res. Natl. Bur. Stand. 45, 239-245 (1950)

21. Varma, S., Taşdelen, F.: Szász type operators involving Charlier polynomials. Math. Comput. Model. 56, 118-122 (2012)

22. Wafi, A., Rao, N., Deepmala: On Kantorovich form of generalized Szász-type operators using Charlier polynomials. Korean J. Math. 25(1), 99-116 (2017). https://doi.org/10.11568/kjm.2017.25.1.99

\section{Submit your manuscript to a SpringerOpen ${ }^{0}$ journal and benefit from:}

- Convenient online submission

- Rigorous peer review

- Open access: articles freely available online

- High visibility within the field

- Retaining the copyright to your article

Submit your next manuscript at $\boldsymbol{~ s p r i n g e r o p e n . c o m ~}$ 